\title{
Aportes de un Curso en Línea Masivo y Abierto (MOOC) sobre la Transferencia del Aprendizaje
}

\author{
Martín A. Mercado-Varela, Ramona I. García-López* y Armando Lozano-Rodríguez \\ Instituto Tecnológico de Sonora, Dpto. de Educación, 5 de febrero 818 sur, Sonora-México \\ (e-mail:martin.mercado@itson.edu.mx; igarcia@itson.edu.mx; armando.lozano@itson.edu.mx) \\ ${ }^{*}$ Autor a quien debe ser dirigida la correspondencia
}

Recibido Ene. 3, 2019; Aceptado Mar. 8, 2019; Versión final Abr. 29, 2019, Publicado Oct. 2019

\begin{abstract}
Resumen
Se discuten y analizan los aportes que puede llegar a tener un Curso en Línea Masivo y Abierto (MOOC, Massive Open Online Course) para la formación docente. Se analizan la construcción de conocimiento y la transferencia del aprendizaje que tiene lugar a partir de la experiencia de los participantes en el curso. Por la vía de la entrevista semiestructurada, se recopila información de 15 docentes que tuvieron una participación activa durante la experiencia formativa. El análisis de contenido es la herramienta para depurar, organizar y analizar la información. Se reportan los aportes sobre el aprendizaje de los diferentes componentes tecnopedagógicos incluidos en la propuesta instruccional del curso, así como diferentes tipos de transferencia dentro la práctica profesional del participante. Se concluye que el MOOC incentiva la transferencia del aprendizaje principalmente al proveer diferentes espacios de interacción entre docentes que se benefician del intercambio intelectual con sus pares.
\end{abstract}

Palabras clave: MOOC; transferencia del aprendizaje; formación docente; componentes tecno-pedagógicos; educación en línea

\section{Contributions of a Massive Open Online Course (MOOC) on the Transfer of Learning}

\begin{abstract}
The contributions that a Massive and Open Online Course (MOOC) can have for teacher training, is discussed and analyzed. The construction of knowledge and the transfer of learning that take place from the experience of the participants in the course are analyzed. Through semi-structured interviews, information is collected from 15 teachers who had active participation during the training experience. The content analysis is the tool to debug, organize and analyze the information. The contributions on the learning process of the different techno-pedagogical components included in the instructional proposal of the course are reported, as well as different types of transfer within the professional practice of the participant. It is concluded that the MOOC encourages the transfer of learning mainly by providing different spaces for interaction between teachers who benefit from intellectual exchange with their peers.
\end{abstract}

Keywords: MOOC; transfer of learning; teacher training; techno pedagogical components; online education 


\section{INTRODUCCIÓN}

Los Cursos en Línea Masivos y Abiertos (MOOC, por su nombre en inglés Massive Open Online Course) irrumpieron en el terreno educativo desde el 2008 con la impartición del curso Conectivismo y Conocimiento Conectivo (CCK08, por su nombre en inglés Connectivism and Connective Knowledge) desarrollado por George Siemens y Stephen Downes auspiciados por la Universidad de Athabasca en Canadá. El curso tenía por objetivo dar a conocer y probar la teoría del Conectivismo concebida por los mismos autores. La integración de ideas relacionadas con la educación abierta, el aprendizaje distribuido y el mismo Conectivismo en la propuesta instruccional del curso, marcó el inicio del formato MOOC (Downes, 2012); o mejor dicho de los cMOOC haciendo la "c" referencia al Conectivismo, en donde la conectividad e interacción entre participantes juegan un rol esencial en los procesos de enseñanza-aprendizaje. Aun con lo innovador de la propuesta no fue hasta el 2012 que este tipo de cursos comenzaron a despertar el interés de investigadores y de la comunidad educativa; en esta etapa se gestó el discurso de los MOOC como una innovación disruptiva capaz de reconfigurar el sistema educativo universitario. Los cursos diseñados a partir de esta nueva etapa se denominan normalmente como xMOOC, los cuales son extensiones de los modelos educativos tradicionales, pero ahora en formato masivo. Los xMOOC dominan el panorama educativo con una cantidad significativa de cursos y millones de aprendices que han experimentado con este formato particular.

Se identifican dos posturas con respecto a los MOOC: 1) relacionada con el acceso a educación de grupos previamente excluidos, la aparición de nuevas pedagogías y la creación de comunidades globales que giran en torno a la educación; 2) vinculada con el asomo de una generación incapaz de comunicarse cara a cara dentro del aula e incluso el fin de la universidad tal y como la conocemos (Breslow et al., 2013). Es así, que el discurso se ha enfocado en ligar a los MOOC con innovaciones en la enseñanza de nivel superior y con la democratización del conocimiento, para acercar la educación de calidad a miles de usuarios que cuenten con un dispositivo y acceso a internet; es debatible si se cumplirán completamente las potencialidades que se le adjudican (Jordan, 2014; Sandeen, 2013). Sin embargo, un modelo que cuestione, complemente o desafíe los modelos existentes de educación siempre es positivo (Mendez, 2013). De esta forma, incorporar innovaciones en el proceso enseñanza-aprendizaje de la educación virtual es una tendencia importante que conlleva a la mejora continua y es primordial para su desarrollo y consolidación.

Aunque la aparición de este formato tiene pocos años se ha desarrollado un considerable cuerpo de literatura. A partir del discurso de los MOOC como innovación disruptiva, numerosas investigaciones se han realizado para informar sobre cuáles son los aportes que brindan a los procesos de enseñanza-aprendizaje. Desde este punto de vista, estos pueden ser vistos como ventanas hacia la innovación, ya que permiten experimentar en formato masivo, la puesta en práctica de diferentes teorías y tendencias educativas. En este sentido, la participación masiva de aprendices hace a los MOOC un espacio para la investigación. Dentro de esta, la formación docente representa una tendencia muy importante, como se ejemplifica en diversas investigaciones (Gynther, 2016; Ji y Cao, 2016; Langset et al., 2018; Laurillard, 2016; Salmon et al., 2015; Vivian et al., 2014; Wambugu, 2018). De acuerdo con Kleiman et al. (2013; 2015), los enfoques de desarrollo profesional son caros, regularmente inefectivos e incapaces de llegar a todos los educadores; se requieren nuevos enfoques escalables, accesibles, sostenibles, y con bajos costos. Al respecto, Silva-Peña y Salgado (2014) mencionan que la demanda por formar docentes es significativa, por lo que los MOOC representan una opción en potencia viable y positiva para formar a gran escala.

Incluso con los avances en materia de formación docente, esta línea de investigación está en procesos de consolidación. Ji y Cao (2016) argumentan que investigar sobre el desarrollo profesional docente a través de MOOC que, si bien se encuentra en una etapa de estudios empíricos, falta por mejorar la calidad de los cursos y lo recursos que se integran en estos para satisfacer la demanda. La literatura que informe sobre los resultados de la puesta en práctica del formato MOOC para realizar formación docente permanece limitada. En este contexto, es necesario seguir experimentando con el formato. Por otro lado, no se encontraron estudios que aborden el tema de la transferencia del aprendizaje, indicador sobre la calidad de este tipo de formación. Transferencia del aprendizaje se define como la efectiva y continua aplicación en el trabajo de habilidades, conocimientos y concepciones logradas en un contexto de desarrollo profesional (De Rijdt et al., 2013). De acuerdo con Blume et al. (2010), dicha transferencia consiste en dos dimensiones: a) generalización, referida al grado en que el conocimiento y habilidades adquiridas en un entorno de aprendizaje se aplican en otros entornos, personas $y / 0$ situaciones de las personas que fueron formadas y b) mantenimiento, hace alusión al grado en que los cambios que resultan de una experiencia de aprendizaje persisten con el tiempo. Aunque la transferencia del aprendizaje sucede en momentos posteriores a una experiencia formativa, sin esta es difícil poder hablar de un proceso educativo de calidad. Al respecto, Baldwin y Ford (1988); Burke y Hutchins (2007); Blume et al. (2010); y De Rijdt et al. (2013), señalan tres factores que se asocian principalmente a la transferencia: 1) las características de los aprendices, 2) el diseño de la formación y 3) el ambiente de trabajo; por lo que es necesario sean tomadas en cuenta en cualquier proceso formativo. 
Por otro lado, la transferencia es una problemática que está presente en todos los modelos de formación, incluso en el presencial. No hay estudios que informen sobre la facilitación de la transferencia en el caso de los MOOC. En este sentido, aún hay mucho por investigar sobre los procesos instruccionales que se desarrollan en dichos cursos, pero principalmente acerca de sus efectos en el aprendizaje. Por lo anterior, es imperante estudiar otras estrategias que coadyuven en la formación docente utilizando MOOC, así como analizar las experiencias de los participantes y sus resultados de aprendizaje. En consecuencia, el presente trabajo se adhiere a la línea de investigación sobre el desarrollo profesional de educadores donde se discuten los resultados de una experiencia de formación docente con el formato MOOC. En particular, se analiza la influencia de los componentes tecno-pedagógicos incluidos en la propuesta instruccional del curso, sobre el aprendizaje y su posterior transferencia; así como los tipos de transferencia logrados por los participantes, en el entendido que tienen una función primordial en la calidad de la formación con MOOC.

\section{METODOLOGÍA}

Se utiliza el método del estudio de caso para realizar los procesos de investigación. Dado que el interés es comprender la construcción de conocimiento y la transferencia del aprendizaje, que tienen lugar en el espacio de un curso MOOC, dicho método tiene un interés instrumental.

\section{Contexto de la investigación}

EI MOOC Innovación Educativa con Recursos Abiertos es la situación educativa analizada, seleccionado principalmente por la oportunidad de aprendizaje que brindaba sobre la formación docente bajo el formato MOOC. El objetivo del curso fue promover el conocimiento sobre los Recursos Educativos Abiertos (REA), los cuales han impulsado exitosamente la idea de que el conocimiento es un activo de la humanidad, por lo que debe ser abierto (Bucarey y Aguilar, 2017). En este sentido, el curso buscaba innovar en procesos formativos a partir de prácticas docentes con la utilización de este tipo de recursos. Los resultados se derivan de su segunda aplicación realizada en septiembre de 2014 por medio de la plataforma Coursera; tuvo una duración de cuatro semanas. El curso se considera un XMOOC ya que presenta una estructura centralizada en una plataforma, aunque también una serie de características como la autogestión, creación de comunidades de aprendizaje y compartición del trabajo intelectual asociadas a los CMOOC. Para mediar el aprendizaje se utilizaron diferentes componentes tecno-pedagógicos: foros de discusión, videos de expertos, REA (lecturas, objetos de aprendizaje, videos etc.), encuestas de opinión, instrumentos de autoevaluación de aprendizajes, evaluación por pares, portafolios electrónicos, grupos de autoestudio, redes sociales y facilitadores. En la Tabla 1 se describe el curso de acuerdo con las dimensiones propuestas por Conole (2013) que tienen presencia en el diseño instruccional en diferentes niveles.

Tabla 1: Características del curso

\begin{tabular}{|l|c|c|c|}
\hline \multirow{2}{*}{\multicolumn{1}{|c|}{ Dimensión }} & \multicolumn{3}{c|}{ Nivel de presencia } \\
\cline { 2 - 4 } & Bajo & Medio & Alto \\
\hline Grado de apertura & & & $\mathrm{X}$ \\
\hline Escala de participación & & & $\mathrm{X}$ \\
\hline Uso de multimedia & & $\mathrm{X}$ & \\
\hline Grado de comunicación & & & $\mathrm{X}$ \\
\hline Grado de colaboración & & & $\mathrm{X}$ \\
\hline Itinerario de aprendizaje (altamente estructurado) & & & $\mathrm{X}$ \\
\hline Aseguramiento de la calidad & & & $\mathrm{X}$ \\
\hline Fomento a la reflexión & & & $\mathrm{X}$ \\
\hline Acreditación & $\mathrm{X}$ & & \\
\hline Grado de formalidad & & $\mathrm{X}$ & \\
\hline Autonomía & & & $\mathrm{X}$ \\
\hline Diversidad & & $\mathrm{X}$ & \\
\hline
\end{tabular}

\section{Participantes}

El curso se impartió en formato abierto alcanzando una matrícula de 14,227 participantes provenientes de 102 países. De acuerdo con los intereses cualitativos de este trabajo, se invitó a colaborar únicamente a participantes con perfil docente pertenecientes a la institución que diseñó el curso, los cuales lo estaban 
cursando como parte de la cuota de formación que les solicita la misma institución. Del grupo de docentes que ingresó, 42 aprobaron el curso, 30 con aprobación normal (entre 60 y 90 de calificación) y 12 con distinción ( $\geq 90$ de calificación). 15 docentes aceptaron colaborar en este trabajo, 11 con aprobación normal y 4 con distinción (Ver Tabla 2). La participación activa para lograr la aprobación sugiere que estuvieron expuestos en gran medida a la propuesta de aprendizaje de la situación educativa. Por lo tanto, esta muestra de docentes aporta información valiosa para el cumplimiento del objetivo de investigación.

Tabla 2: Características de los participantes

\begin{tabular}{|c|c|l|l|}
\hline Clave & Sexo & \multicolumn{1}{|c|}{ Área laboral } & Tipo de aprobación \\
\hline D1 & H & Ciencias & Normal \\
\hline D2 & H & Relaciones internacionales & Normal \\
\hline D3 & M & Innovación & Normal \\
\hline D4 & M & Lenguas modernas & Normal \\
\hline D5 & M & Ingeniería & Normal \\
\hline D6 & M & Matemáticas & Normal \\
\hline D7 & H & División de profesional & Normal \\
\hline D8 & M & Ciencias & Normal \\
\hline D9 & M & Comunicación y humanidades & Distinción \\
\hline D10 & M & Ciencias & Normal \\
\hline D11 & M & División preparatoria & Distinción \\
\hline D12 & H & Ingeniero mecánico administrador & Normal \\
\hline D13 & H & Biotecnología & Distinción \\
\hline D14 & M & Mercadotecnia & Distinción \\
\hline D15 & M & División de profesional & Normal \\
\hline
\end{tabular}

\section{Categorías de estudio}

Las categorías representan las áreas del fenómeno a indagar: 1) construcción de conocimiento que se define como las formas en que se construye conocimiento a través de los componentes tecno-pedagógicos (plataforma, contenidos, aplicaciones tecnológicas, actividades de aprendizaje, y espacios de socialización) de un ambiente de aprendizaje masivo, que en conjunto integran el formato y propuesta de aprendizaje (Alario et al., 2014; Glance et al., 2013; Siemens, 2013; Spyropoulou et al., 2014); al interior de la categoría se analizan los códigos procesos de socialización: formas y medios de comunicación por los que establecen contacto los participantes, lo que facilita e implica distintos tipos de intercambios intelectuales (ideas, metodologías, recurso, etc.) y prácticas en la construcción de conocimiento: actividad del participante que es mediada por los componentes tecno-pedagógicos del curso (materiales, actividades, espacios de socialización, etc.) en la construcción de sus trayectos y redes de aprendizaje. 2) Transferencia del aprendizaje entendida como la aplicación continua de conocimientos obtenidos en un contexto de formación al contexto profesional docente, lo que representa cambios en las prácticas docentes (Baldwin y Ford, 1988; De Rijdt et al., 2013); al interior de la categoría, se analizan los códigos tipos de transferencia: distintos tipos de conocimiento que logran ser aplicados de un contexto a otro y factores de transferencia: características individuales del participante, del curso y del contexto, que median la aplicación de conocimiento de un contexto a otro.

\section{Instrumentos}

Se aplicó una entrevista semiestructurada en los formatos cara a cara y en línea para recuperar información de los participantes, considerando que, aunque los participantes son parte de la misma institución, no trabajan en el mismo campus ni en la misma región del país. Los sistemas por conferencias Skype y Google Hangout fueron las herramientas para las entrevistas en línea. La entrevista consistía en un conjunto de 20 preguntas, las cuales se intercalaban dependiendo la conveniencia durante la plática. En ocasiones, no se realizaba el total de preguntas, esto dependía de la profundidad de las respuestas del entrevistado.

La aplicación tuvo lugar aproximadamente un año después de la impartición de la situación educativa; inició en diciembre de 2015 y terminó en marzo de 2016. Esta decisión está relacionada con la medida de transferencia; como sugiere la literatura, se dio la oportunidad al participante (en términos de tiempo) de transferir lo aprendido en el curso en su contexto profesional. Se realizaron 15 en total, una por cada docente, obteniendo 05:07:48 (hh:mm:ss) de tiempo de grabación. 


\section{Análisis de los datos}

Se utilizó el software para análisis de datos cualitativos y métodos mixtos MAXQDA, privilegiando el análisis de contenido. Esto permitió la visualización de los datos (extractos de texto codificados), lo que facilitó el análisis al poder identificar en una sola imagen los códigos utilizados, la secuencia de codificación, la magnitud de cada código, así como el "noise" (extractos de texto no codificados); además, permitió obtener una matriz de códigos (frecuencias de aparición) para poder agrupar las categorizaciones y facilitar el análisis de datos de las entrevistas.

En particular, sobre el análisis, para dar confiabilidad a los resultados y conclusiones, se realizó un ejercicio entre codificadores que consistió en categorizar una muestra (10\%) de los datos, con el objetivo de unificar criterios al momento de clasificar determinados extractos de texto. Una vez unificados los criterios de los codificadores, se procedió a categorizar la totalidad de los datos, lo que posteriormente dio paso al análisis. El análisis de las categorías 1) construcción de conocimiento y 2) transferencia del aprendizaje tuvo por objetivo comprender cuáles son los componentes tecno-pedagógicos que tienen una mayor influencia en el aprendizaje y que facilitan su transferencia al contexto profesional de los participantes en momentos posteriores, así como los tipos de transferencia. Por lo tanto, para orientar el análisis e interpretación de los datos de las categorías, se plantearon dos interrogantes:

¿Cuál es el aporte de los componentes tecno-pedagógicos sobre el aprendizaje y su posterior transferencia?

¿Qué tipos de transferencia tuvieron lugar a partir de la experiencia de los docentes en el curso?

\section{RESULTADOS Y DISCUSIÓN}

Los resultados se presentan por categorías y se acompañan de citas textuales de los entrevistados para dar evidencias de validez. Son consecuencia, de la categorización y análisis de 243 extractos de texto recuperados de las entrevistas con los docentes y, que reflejan la interpretación sobre lo acontecido durante y posterior al curso; 140 pertenecen a la categoría Construcción de conocimiento y 103 a la de Transferencia del aprendizaje, tal como se muestra en la Figura 1.

Categoría 1: Construcción de conocimiento

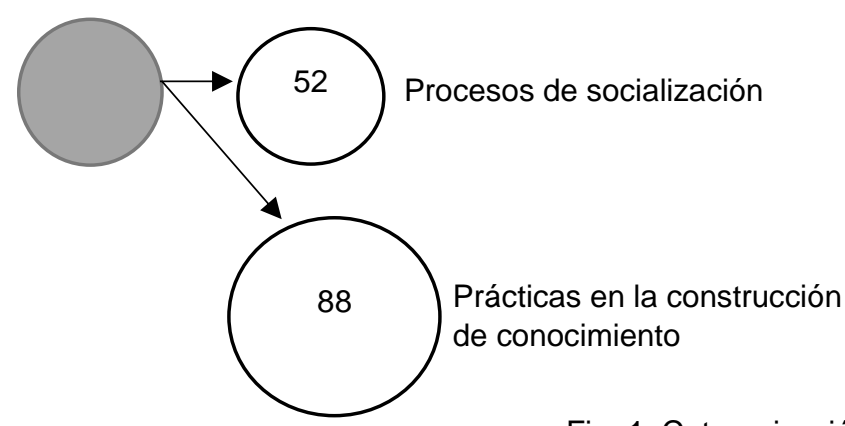

Fig. 1: Categorización de datos

\section{Categoría 2: Transferencia del aprendizaje}

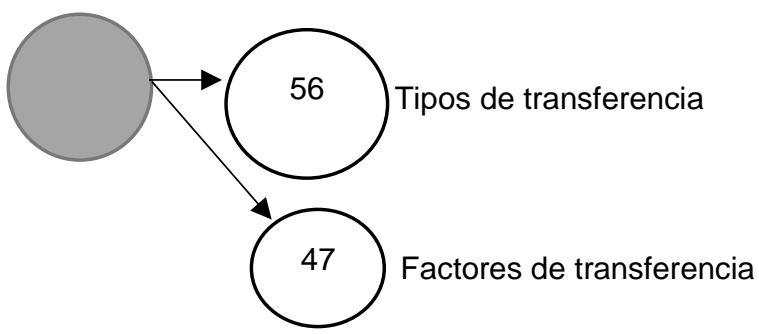

\section{Construcción de conocimiento}

Los docentes mencionaron los significados que le otorgan a los componentes tecno-pedagógicos que favorecen el aprendizaje y la transferencia, principalmente de los que tenían por objetivo promover la interacción entre participantes. Señalaron que los videos de expertos, los foros de discusión y la evaluación entre pares, son los principales elementos que contribuyen a tan importante función.

En ese sentido, los videos de expertos fueron los más valorados en relación con el aprendizaje. Dicha valoración se presentó en tres aspectos: 1) como recurso en sí mismo, debido a su especialización, experiencia del expositor, las directrices para la realización de actividades, promover la reflexión, la incorporación de recursos multimedia; 2) su influencia en diversos momentos del proceso educativo, pues se usaron al inicio y para el desarrollo de actividades, así como para guiar y contextualizar el aprendizaje; y 3) como componente social, al referirlo como promotor del contacto humano y aprender de la voz e imagen de las personas. Algunas de las opiniones de los entrevistados fueron: D14: "Los videos los tengo descargados, ¡todos! [...] era material muy interesante, pero como dice un dicho: 'nada impacta más a un ser humano que otro ser humano". D2: "[...] tenían como objetivo, pues guiar un poco la reflexión y la manera en como teníamos que entregar los productos [...] realmente la herramienta más importante que me ha permitido 
construir este camino". D15: "Me gustaron mucho los videos sobre todo el contacto con los expertos [...] muy importante cuando se está trabajando así a distancia, porque como que es importante ver al final de cuentas el contacto humano".

Como recurso valioso para fomentar la socialización, los docentes consideraron como principal e importante a los foros de discusión, aun por arriba de Twitter y Facebook. Lo anterior, debido a la interacción que generaron entre todos los participantes; esto al compartir ideas, métodos, materiales, experiencias, prácticas docentes y emociones, lo que enriqueció el aprendizaje y favoreció la transferencia. Los siguientes comentarios avalan lo dicho anteriormente:

D9: "Pues indiscutiblemente la participación en los foros, el ver cómo trabajan otros colegas, otros docentes, en escuelas públicas, en escuelas tal vez privadas pero en otras ciudades y hasta en otros países [...] entonces todas esas ideas que [...] yo pude ir rescatando poco a poco, enriquecían mi manera de trabajar en cada actividad [...] aunque los contenidos te aportan muchísima información pues no hay como el sentir de tu colega, que está en otra ciudad, en otro país, en otro tipo de institución educativa, eso enriquece mucho. Siempre tenía comentarios de motivación y aplaudía las ideas que me parecían importantes, entonces, eso yo creo que te motiva a seguir y a seguir participando y pues a no abandonar el curso [hace referencia a la socialización]". D2: "Sí se me hace muy relevante, muy importante tener esta interacción, construye finalmente una red de manera ¿verdad? Horizontal, o tener esta percepción de que estamos en un espacio horizontal no vertical". D10: "Estos foros que... estaban dentro de la propia plataforma [...] estos espacios me sirvieron [...] no fue tanto para socializar el conocimiento pretendido sino más bien para socializar la emoción, la frustración, la satisfacción, las dudas, la información que se estaba dando en estos espacios".

En cuanto a la evaluación de pares puede decirse que es una estrategia que permite que el participante se responsabilice de su propio aprendizaje y el de sus compañeros; ya que fomenta la comunicación mediante las realimentaciones que se dan a las actividades, así como al compartir materiales y prácticas docentes; todo orientado a fortalecer el aprendizaje y promover su transferencia. Al respecto, uno de los participantes señaló: D3: "[...] te permite ver, darte cuenta de otras perspectivas... retroalimentar y dar, obviamente tus comentarios sobre tu perspectiva y recibir también otras opiniones, ver cosas que, pues no, pues ni siquiera se te hubieran ocurrido y pues eso da pie a... pues a mejorar, a mejorar tus formas de... impartir clases [...] tus actividades [...] me ayudó a ver otras perspectivas que yo no había considerado, ni siquiera me había pasado por la cabeza, ni me había preguntado".

Cabe señalar que la interacción (acercamiento) entre los participantes facilitó el desarrollo del aprendizaje y su transferencia al ámbito profesional; esto debido a que tuvieron la oportunidad de intercambiar diferentes perspectivas sobre sus propias prácticas docentes, lo que favoreció que conocieran nuevas formas de aplicar el conocimiento, principalmente lo relacionado con el uso de REA dentro del proceso educativo. No obstante, aunque un gran número de docentes señaló a la interacción como un elemento importante; otro grupo más reducido opinó lo contrario, resaltando que no se requiere dicha interacción para aprovechar el curso, ya que de por sí es complicado contactar a los demás por la comunicación asincrónica, el número de foros e incluso lo impersonal en los comunicados escritos. Al respecto, un docente puntualizó lo siguiente:

D5: "Precisamente fue en la parte que más se me complicó, el elegir tu par [...] Entonces es un mundo de gente participando [...] yo no lo sentí tan necesario, eh, porque al final de cuenta tú logras hacer las actividades, tengas o no tengas un par; si cuando estabas frente a la persona es difícil trabajar con ella, imagínate cuando no coinciden en horario, cuando no hay disponibilidad de alguna de las partes, esto se complica aún más".

De lo anterior se puede desprender que algunos participantes siguen teniendo influencia de los modelos presenciales o de modelos en línea ya conocidos, en donde se privilegia la comunicación sincrónica con los mismos participantes. Hubo varios señalamientos sobre lo complicado que resulta participar en un espacio virtual donde impera la teoría Conectivista, cuya premisa básica es la interacción como instrumento de aprendizaje. Desde esta teoría, el conocimiento está repartido en espacios distintos, en donde se incluyen los dispositivos no humanos, como lo son las bases de datos o las diferentes alternativas que ofrece el internet. Aprender en este contexto involucra conectar otros participantes mediante esos espacios diversos.

\section{Transferencia del aprendizaje}

La transferencia del aprendizaje se ubicó en los entornos profesionales de los docentes. Un hallazgo que se resalta es que los conocimientos transferidos por estos últimos tienen que ver con la producción, búsqueda, selección y movilización de REA, que se alinean a las competencias del curso. En relación con lo anterior, un docente señaló: D10: "Una es la conciencia de la existencia de este tipo de recursos [hace referencia a los REA], entonces nos implica que la mayoría de las veces, antes de comenzar a generar un material 
completamente nuevo acudo a estos otros recursos para ver si lo puedo tropicalizar, es decir, si puedo echar mano de él, y si de esta forma lo enriquezco, lo traduzco o me da una idea de cómo hacer el mío. Entonces... es ya como una práctica no generar algo totalmente nuevo sino explorar si hay algo existente de la calidad que yo deseo y de esta manera me ahorro trabajo".

Lo anterior supone que el participante puede evaluar de manera crítica un REA, lo que se traduce en un conocimiento de los usos que puede tener el recurso con base en su tipo de licencia. También es posible evidenciar la generalización (aplicar en un contexto distinto lo que se ha aprendido previamente) y el mantenimiento (la posibilidad de seguir aplicando) de los conocimientos adquiridos en el curso. Aunado a lo anterior, la cultura de los REA entre los participantes fue fomentada porque algunos de ellos reconocen la importancia de la educación abierta en el aprendizaje: D7: "[...] de hecho yo ya conocía Coursera, ya había estado tomando algunas capacitaciones antes con algunas otras universidades, pero cuando yo estudié el curso de REA me permitió entender cómo funcionaban las plataformas [hace referencia a plataformas abiertas] y sobre todo entender que la educación va en ese sentido, que es algo bastante complicado porque está el asunto de los costos de por medio [...] Tenemos que cambiar los métodos de trabajo y de educación, porque con tantos modos de educación abierta, ya los modelos de las universidades están siendo rebasados".

También, se resalta el valor pedagógico de los REA al realizar prácticas pedagógicas con sus propios alumnos, tal y como lo señaló un docente: D8: "[...] nosotros ahorita ya de manera cotidiana metemos a los chicos en ciertos recursos [sic] que hemos ido encontrando, que nos proporcionaron ahí en el curso y que nosotros hemos ido agregando; los tenemos ya como fijos, como material fijo para que los chicos trabajen". D10: "[...] ciertos cursos en los que yo exploro hay ciertas lecciones que están ahí grabadas, con transcripciones incluso, y a partir de este material yo realizo ejercicios para mis estudiantes, entonces lo que decía, exploro en [...] universidades de Estados Unidos para ver si hay cursos parecidos a los míos, o muchas veces no es el curso exacto, pero hay ciertas unidades que me pueden servir [...] esto es algo que a partir del curso que tomé ha seguido como una práctica".

De hecho, la cultura MOOC fue fomentada en algunos docentes, pues los han utilizado como recursos de aprendizaje en su propia práctica docente, realizando actividades con sus alumnos y como opción para su formación. Algunos docentes señalaron: D11: "[...] a raíz de ese curso [hace referencia al curso], yo con mis alumnos manejo el que ellos en el semestre mínimo se tienen que meter a un curso, generalmente es de Coursera y de los del Tec de Monterrey, para que ellos también vayan aprendiendo a aprender, a través de justamente de este tipo de herramientas, es algo de lo que se ha ido metiendo a los cursos y de alguna manera hacemos que ellos también empiecen a buscar... les doy una pauta de entre qué temas y qué temas para darles una amplitud y ya ellos van escogiendo lo que a ellos les llame más la atención [...]" D5: "Sí, fue cuando me empecé a familiarizar [hace referencia a cuando a empezó a buscar REA dentro del curso] y a darme cuenta que esto es, tiene un potencial enorme, yo he seguido entrando a Coursera y tomando uno que otro curso de este tipo, de los gratis que ofrece".

Hubo dos factores de la situación educativa que promovieron la transferencia del aprendizaje; la organización del curso (diseño de intervención) y las interacciones que se dieron en los espacios de socialización. Al inicio, la organización del curso dio paso al desarrollo de actividades de aprendizaje por parte de los participantes. Un docente señaló: D1: "Creo que algo que me ayudó muchísimo, fue la manera en como estaba organizado el curso [...] estaba muy bien estructurado en cuanto a calendario de actividades [...] la misma manera en como estaba diseñado el curso, te obliga a... [...] autogestionarte, organizarte [...] eso después pues lo reflejas a la hora que estás preparando material, información para tus grupos [...] y ya esa autogestión que vas haciendo muy consciente durante el desarrollo, luego la quieres aplicar en la organización de tus demás cursos".

Posteriormente, el intercambio de ideas y de recursos didácticos disponibles en los espacios de socialización provocó la transferencia del aprendizaje. Al respecto, un docente comentó: D2: "[...] el curso, de manera general pues... lo vi muy bien organizado, muy bien estructurado, la metodología me pareció muy adecuada [...] y lo más interesante para mí como profesor investigador, es que sí ha ido impactando, digamos al nivel de la metodología ¿no?, la manera como... impactó mis materias, sobre todo en el contacto que tengo tanto con los estudiantes como con la sociedad civil [...] en nuestro contexto mexicano que sufrimos de [...] parroquialismo académico se me hace muy, muy valioso, digamos, tener este tipo de interacción ¿no?, entre colegas de diferentes instituciones públicas y privadas, sí, sí, opinas, pues este, sobre la metodología del otro $[\ldots]$ cuestionar o lo que sea $[\ldots]$..

Se reconoce que la configuración de un MOOC puede variar al integrar diferentes componentes tecnopedagógicos en la propuesta de aprendizaje. Sin embargo, para facilitar el aprendizaje y una eventual transferencia, la elección de un diseño de formación orientado hacia la interacción entre docentes es fundamental. Por otro lado, la influencia de los modelos educativos tradicionales, tanto presenciales como en 
línea, puede desvirtuar la interacción entre participantes que tiene lugar en dichos cursos, la cual es uno de los componentes más importantes en los procesos de enseñanza-aprendizaje dentro de estos espacios educativos. En la Figura 2 se ilustra un resumen de los hallazgos de este trabajo: la interacción de los participantes con los diferentes componentes tecno-pedagógicos del curso (videos de expertos, evaluación por pares y foros de discusión) contribuyó en un primer momento a lograr los objetivos de aprendizaje, principalmente por el acercamiento y compartición de recursos entre participantes. En un segundo momento, precisamente el acercamiento y compartición de recursos (emociones, perspectivas, experiencias etc.) permitió que los docentes conocieran diferentes prácticas de sus pares, así como nuevas formas de aplicación del conocimiento. En esta investigación señalamos dos tipos de transferencia, Cultura MOOC y Cultura REA, que describen la visión y las prácticas que los docentes realizan en su contexto profesional con ambos recursos.

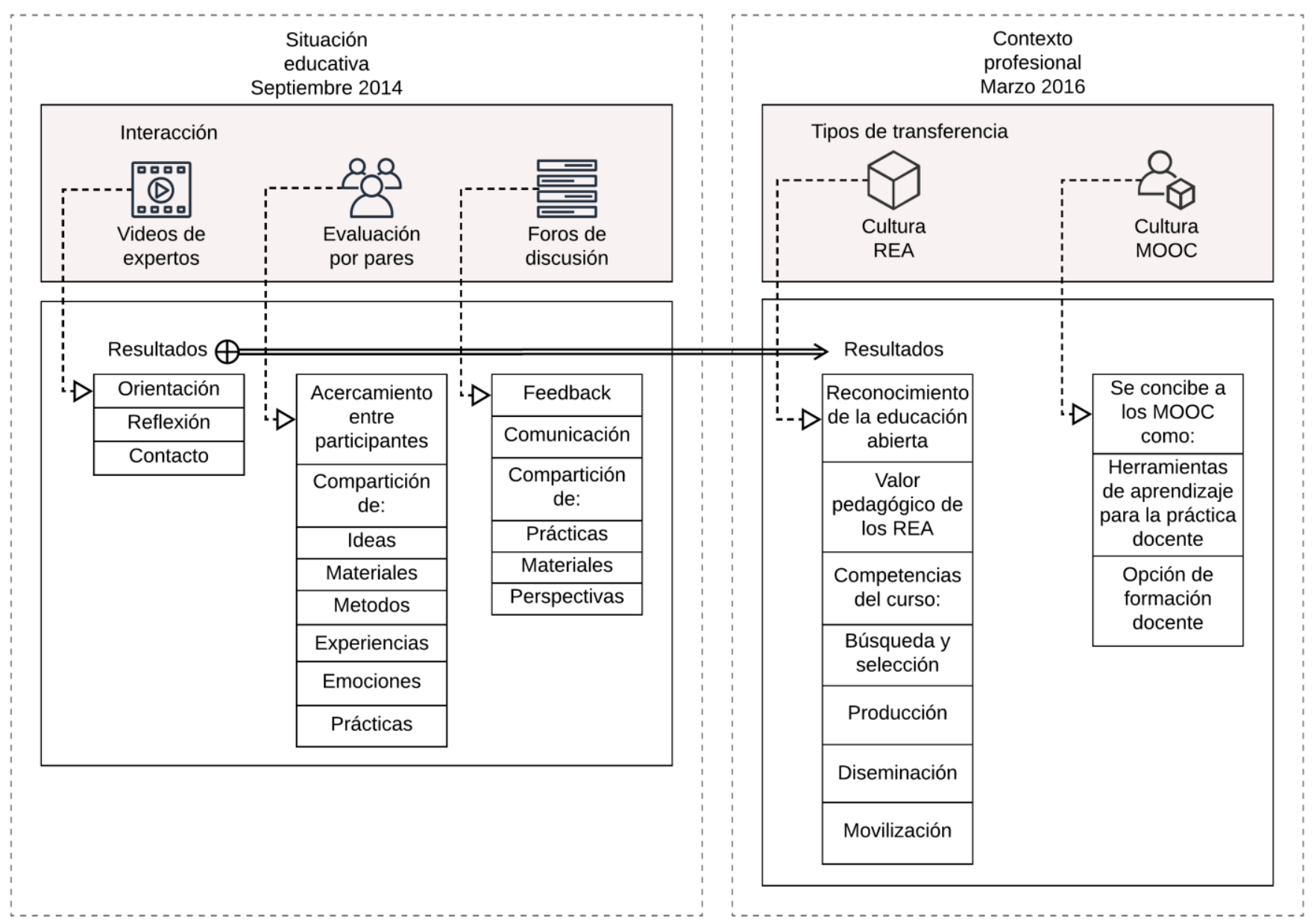

Fig. 2: Resumen de hallazgos

\section{CONCLUSIONES}

Derivado del análisis de los resultados del caso analizado, se extraen cuatro conclusiones principales, respecto a los aportes de un MOOC sobre la transferencia del aprendizaje: 1) componentes tecnopedagógicos como los videos de expertos, evaluación por pares, y foros de discusión, facilitan el aprendizaje y posteriormente su transferencia al contexto profesional; 2) el atributo social de los foros de discusión permite el acercamiento e intercambio intelectual entre docentes; 3 ) un diseño de formación orientado hacia la interacción contribuye a la transferencia del aprendizaje al poner de relieve entre los docentes, diferentes formas de aplicación del conocimiento y; 4) se pueden lograr distintos tipos de transferencia al participar en un MOOC enfocado en el uso de REA: en particular, la que se nombra como cultura MOOC, que se define como la predisposición de utilizarlos como una herramienta de aprendizaje para la práctica docente y como una opción de formación continua. De esta forma, se les concibe como un recurso de aprendizaje complementario para la práctica docente; lo que evidencia flexibilidad para apoyar otros tipos de formación, independientemente que representen además un formato de formación particular.

\section{REFERENCIAS}

Alario-Hoyos, C.A., M. Perez-Sanagustín y otros cuatro autores, Designing Your First MOOC from Scratch: Recommendations after Teaching "Digital Education of the Future", eLearning papers, ISSN: 1887-1542, (37), (2014) 
Baldwin, T. y K. Ford, Transfer of Training: A Review and Directions for Future Research, doi: 10.1111/j.17446570.1988.tb00632.x, Personnel Psychology, 41(1), 63-105 (1988)

Blume, B., K. Ford, T. Baldwin y J. Huang, Transfer of Training: A Meta-analytic Review, doi: 10.1177/0149206309352880, Journal of Management, 36(4), (2010)

Breslow, L., D. Pritchard, J. DeBoer, G. Stump y otros dos autores, Studying Learning in the Worldwide Classroom, Research into edX's First MOOC, Research \& Practice in Assessment, ISSN: 2161-4210, 8, 13-25 (2013)

Bucarey, S. y M. Aguilar, Recursos Educativos Abiertos en la Facultad de Medicina de la Universidad Austral de Chile, Proyecto AUS1410, http://dx.doi.org/10.4067/S0718-50062017000200004, Form. Univ., 10(2), 23-30 (2017)

Burke, L. y H. Hutchins, Training Transfer: an Integrative Literature Review, doi: 10.1177/1534484307303035, Human Resource Development Review, 6, 263-296 (2007)

Cohen, L., L. Manion y K. Morrison, Research Methods in Education, 6ª Ed., Routledge, USA (2007)

Conole, G., Los MOOC como Tecnologías Disruptivas: Estrategias para Mejorar la Experiencia de Aprendizaje y la Calidad de los MOOC, Campus Virtuales, ISSN: 2255-1514, 2(2), 16-28 (2013)

De Rijdt, C., A. Stes, C. Van der Vleuten y F. Dochy, Influencing Variables and Moderators of Transfer of Learning to the Work Place within the Area of Staff Development in Higher education: Research Review, doi: 10.1016/j.edurev.2012.05.007, Educational Research Review, 8, 48-74 (2013)

Downes, S., The Rise of MOOCs. gRSShopper, Canadá, (2012)

Glance, D., M. Forsey y M. Riley, The Pedagogical Foundations of Massive Open Online Courses, Peer-reviewed Journal of Internet, ISSN: 1396-0466, 18(5), (2013)

Gynther, K., Design Framework for an Adaptive MOOC Enhanced by Blended Learning: Supplementary Training and Personalized Learning for Teacher Professional Development, The Electronic Journal of e-learning, ISSN: 1479-4403, 14(1), 15-30 (2016)

Ji, Z., e Y. Cao, A Prospective Study on the Application of MOOC in Teacher Professional Development in China, doi: 10.13189/ujer.2016.040917, Universal Journal of Educational Research, 4(9), 2061-2067 (2016)

Jordan, K., Initial Trends in Enrolment and Completion of Massive Open Online Courses, http://dx.doi.org/10.19173/irrodl.v15i1.1651, Inter. Review of Research in Open and Distance Learning, 15(1), 134-160 (2014)

Kleiman, G., M. Wolf y D. Frye, Educating Educators: Designing MOOC for Professional Learning; in P. Kim. Massive Open Online Courses: the MOOC Revolution, Routledge, 117-145, New York (2015)

Kleiman, G., M. Wolf y D. Frye, The Digital Learning Transition MOOC for Educators: Exploring a Scalable Approach to Professional Development, Friday Institute, NC State University (2013)

Langset, I., D. Jacobsen y H. Haugsbakken, Digital Professional Development: Towards a Collaborative Learning Approach for Taking Higher Education into the Digitalized Age, doi: 10.18261/ISSN.1891-943X-2018-01-03, Nordic Journal of Digital Literacy, 13(1), 24-39 (2018)

Laurillard, D., The Educational Problem that MOOC Could Solve: Professional Development for Teachers of Disadvantaged Students, doi: 10.3402/rlt.v24.29369, Research in Learning Technology, 24(1), 1-24 (2016)

Méndez, C., Diseño e Implementación de Cursos Abiertos Masivos y en Línea (MOOC): Expectativas y Consideraciones Prácticas, Revista de Educación a Distancia, ISSN: 1578-7680, (39), 58-77 (2013)

Salmon, G., J. Gregory, K. Lokuge y B. Ross, Experiential Online Development for Educators: The Example of the Carpe Diem MOOC, doi: 10.1111/bjet.12256, British Journal of Educational Technology, 46(3), 542-556 (2015)

Sandeen, C., Assessment's Place in the New MOOC World, Research \& Practice Assesment, ISSN: 2161-4210, 8, 5-12 (2013)

Siemens, G., Massive Open Online Courses: Innovation in education?; in R. McGreal, W. Kinuthia and S. Marshall. Open Educational Resources: Innovation, Research and Practice, Commonwealth of Learning and Athabasca University, 5-16, Vancouver (2013)

Silva-Peña, I. e I. Salgado, Utilización de MOOC en la Formación Docente: Ventajas, Desventajas y Peligros, Revista de Currículum y Formación del Profesorado, ISSN: 1138-414X,18(1), 155-166 (2014)

Spyropoulou, N., C. Pierrakeas y A. Kameas, Creating mooc guidelines based on best practices; Actas de 6th International Conference on Education and New Learning Technologies, 6981-6990, Barcelo, España, 7-9 de Julio (2014)

Vivian, R., K. Falkner y N. Falkner, Addressing the Challenges of a New Digital Technologies Curriculum: MOOC as a Scalable Solution for Teacher Professional Development, doi: 10.3402/rlt.v22.24691, Research in Learning Technology, 22 (2014)

Wambugu, P. W., Massive Open Online Courses (MOOC) for Professional Teacher and Teacher Educator Development: A Case of TESSA MOOC in Kenya, doi: 10.13189/ujer.2018.060604, Universal Journal of Educational Research, 6(6), 1153-1157 (2018) 
